\title{
Video Article \\ Fluorescent Nanoparticles for the Measurement of Ion Concentration in Biological Systems
}

\author{
J. Matthew Dubach ${ }^{1}$, Mary K. Balaconis ${ }^{1}$, Heather A. Clark ${ }^{2}$ \\ ${ }^{1}$ Bioengineering Department, Northeastern University \\ ${ }^{2}$ Department of Pharmaceutical Sciences, Northeastern University
}

Correspondence to: Heather A. Clark at h.clark@neu.edu

URL: https://www.jove.com/video/2896

DOI: doi:10.3791/2896

Keywords: Bioengineering, Issue 53, nanoparticles, nanosensors, polymer, fluorescence, imaging, intracellular, in vivo, sodium

Date Published: 7/4/2011

Citation: Dubach, J.M., Balaconis, M.K., Clark, H.A. Fluorescent Nanoparticles for the Measurement of lon Concentration in Biological Systems. J. Vis. Exp. (53), e2896, doi:10.3791/2896 (2011).

\section{Abstract}

Tightly regulated ion homeostasis throughout the body is necessary for the prevention of such debilitating states as dehydration. ${ }^{1}$ In contrast, rapid ion fluxes at the cellular level are required for initiating action potentials in excitable cells. ${ }^{2}$ Sodium regulation plays an important role in both of these cases; however, no method currently exists for continuously monitoring sodium levels in vivo ${ }^{3}$ and intracellular sodium probes ${ }^{4}$ do not provide similar detailed results as calcium probes. In an effort to fill both of these voids, fluorescent nanosensors have been developed that can monitor sodium concentrations in vitro and in vivo. ${ }^{5,6}$ These sensors are based on ion-selective optode technology and consist of plasticized polymeric particles in which sodium specific recognition elements, $\mathrm{pH}$-sensitive fluorophores, and additives are embedded. ${ }^{7-9}$ Mechanistically, the sodium recognition element extracts sodium into the sensor. ${ }^{10}$ This extraction causes the $\mathrm{pH}$-sensitive fluorophore to release a hydrogen ion to maintain charge neutrality within the sensor which causes a change in fluorescence. The sodium sensors are reversible and selective for sodium over potassium even at high intracellular concentrations. ${ }^{6}$ They are approximately $120 \mathrm{~nm}$ in diameter and are coated with polyethylene glycol to impart biocompatibility. Using microinjection techniques, the sensors can be delivered into the cytoplasm of cells where they have been shown to monitor the temporal and spatial sodium dynamics of beating cardiac myocytes. ${ }^{11}$ Additionally, they have also tracked realtime changes in sodium concentrations in vivo when injected subcutaneously into mice. ${ }^{3}$ Herein, we explain in detail and demonstrate the methodology for fabricating fluorescent sodium nanosensors and briefly demonstrate the biological applications our lab uses the nanosensors for: the microinjection of the sensors into cells; and the subcutaneous injection of the sensors into mice.

\section{Video Link}

The video component of this article can be found at https://www.jove.com/video/2896/

\section{Protocol}

\section{Preparation of optode}

Before making the optode, aliquots of the components are need so that they can be easily measured and stored.

1. A $50 \mathrm{mg}$ Sodium lonophore $X(\mathrm{NalX})$ vial and a $50 \mathrm{mg}$ sodium tetrakis[3,5-bis(trifluoromethyl)phenyl]borate (NaTFPB) will each be brought up in tetrahyrdofuran (THF) and aliquoted into $1.5 \mathrm{ml}$ polystyrene centrifuge tubes. In a chemical fume hood, dissolve the $50 \mathrm{mg}$ of NalX in $1 \mathrm{ml}$ of THF in the shipping vial and mix to ensure that the solid has completely dissolved. Transfer $100 \mu \mathrm{l}$ of this solution into 10 separate centrifuge tubes. Repeat for NaTFPB. Allow the THF to evaporate in a chemical fume hood overnight, label the centrifuge tubes and place them in a 4 degree refrigerator for storage. This creates $5 \mathrm{mg}$ aliquots of dry NalX and NaTFPB.

2. Dissolve the Chromoionophore III (CHIII) in $1 \mathrm{ml}$ of THF and transfer to a $3 \mathrm{ml}$ glass vial. Add another $1 \mathrm{ml}$ of THF to the $\mathrm{CHIII}$ shipping vial to dissolve any residual $\mathrm{CHIII}$ and add this to the $3 \mathrm{ml}$ glass vial. There will be a total of $2 \mathrm{ml}$ now. Transfer $1 \mathrm{ml}$ of the CHIII in THF solution to two separate $1.5 \mathrm{ml}$ glass vials with a Teflon coated caps. Store the $\mathrm{CHIII}$ solution in a 4 degree refrigerator at the final concentration of $5 \mathrm{mg} /$ $\mathrm{ml}$.

These aliquots and solutions will be used to make the optode material.

3. Pipette $66 \mu \mathrm{l}$ of bis(2-ethylhexyl)sebacate (DOS) into a $1.5 \mathrm{ml}$ glass vial with a Teflon coated screw cap - this is the optode vial. DOS is a viscous solution so care must be taken to ensure the proper amount of solution is transferred to the vial. The volume corresponds to $60 \mathrm{mg}$ of DOS.

4. Weigh out $30 \mathrm{mg}$ of high molecular weight poly(vinyl) chloride (PVC) and transfer this to the optode vial.

5. Now add the aliquoted functional components to the optode vial to create the desired optode. The relative concentrations of $\mathrm{CHIII}$, NalX, and NaTFPB will determine the response of the sensor to sodium. These concentrations can be adjusted to have a sensor that ideally responds to intracellular concentrations, with a Kd of $10 \mathrm{mM}$, or extracellular concentrations, with a $\mathrm{Kd}$ of $150 \mathrm{mM}$. Additionally, there will likely be 
batch to batch variability of the chemicals from the vendor and calibration of the sensors is necessary to determine if the correct response is occurring for each new batch of chemical components. Here we describe the method to create a sensor with concentrations that are typically used for intracellular sodium measurements.

6. In a chemical fume hood, transfer $100 \mu \mathrm{l}$ of the $5 \mathrm{mg} / \mathrm{ml} \mathrm{CHIII} \mathrm{in} \mathrm{THF} \mathrm{solution} \mathrm{to} \mathrm{the} \mathrm{optode} \mathrm{vial.}$

7. Dissolve $5 \mathrm{mg}$ of $\mathrm{NalX}$ in $300 \mu \mathrm{l}$ of THF and transfer $300 \mu \mathrm{l}$ to the optode vial, this correlates to $5 \mathrm{mg}$ of $\mathrm{NalX}$.

8. Dissolve a $5 \mathrm{mg}$ aliquot of the NaTFPB in $500 \mu \mathrm{l}$ of THF and transfer $20 \mu \mathrm{l}$ of the solution to the optode vial, this correlates to $0.1 \mathrm{mg}$ of NaTFPB.

9. Add $80 \mu \mathrm{l}$ of THF to the optode vial.

10. The solution in the optode vial contains $30 \mathrm{mg}$ of PVC, $60 \mathrm{mg}$ of DOS, $5 \mathrm{mg}$ of NalX, $0.2 \mathrm{mg}$ of NaTFPB, $0.5 \mathrm{mg}$ of CHIII in $500 \mu \mathrm{l}$ of THF. Gently vortex this vial until all of the PVC has dissolved. The optode should be a red color. The total amount of THF added to the vial should be $500 \mu \mathrm{L}$ regardless of the ratio of components used.

11. Allow the remaining THF in the NaIX and NaTFPB aliquots to evaporate overnight and re-label the tube with the correct amount of chemical.

\section{Creating nanosensors}

1. Pipette $100 \mu \mathrm{l}$ of $10 \mathrm{mg} / \mathrm{ml}$ 1,2-Distearoyl-sn-Glycero-3-Phosphoethanolamine-N -[Methoxy(Polyethyleneglycol)-550] in chloroform (PEG-lipid) into a 4 dram glass vial. Allow the chloroform to evaporate in a chemical fume hood. This process can be expedited by drying the solution with nitrogen gas or house air.

2. Add $4 \mathrm{ml}$ of the desired aqueous solution to the vial with PEG-lipid. Place the vial on a laboratory jack underneath the sonicating tip and raise the vial until the tip is about $7 \mathrm{~mm}$ deep in the solution and sonicate at a low sonication power for 30 seconds. Here we use a Branson digital sonifier set to $15 \%$ amplitude with a $1 / 2$ inch tip diameter step horn. The aqueous solution can be any solution. For example, to determine the response of the sensors we use $10 \mathrm{mM}$ HEPES pH 7.2 with trizma base.

3. While the solution is being sonicated, mix $50 \mu \mathrm{l}$ of the optode material with $50 \mu \mathrm{l}$ of dichloromethane in a $0.6 \mathrm{ml}$ microcentrifuge tube. Mix with the pipette to ensure even mixing.

4. Adjust the sonifier control to sonicate for 3 minutes. Start the sonication and while sonicating add the optode mixture solution to the vial by immersing the pipette tip into the solution and dispensing the $100 \mu \mathrm{l}$ of solution in one quick, even injection. The solution should turn blue, depending on the aqueous solution used.

5. Allow the sonication to continue for about 30 seconds then lower the jack so that the sonicating tip is about $2 \mathrm{~mm}$ deep in solution. This should begin to aerate the solution and the solution will become opaque. This step helps to remove the residual THF and dichloromethane from the solution.

6. Once sonication is complete, pull up the nanosensor solution into a $5 \mathrm{ml}$ syringe. Attach a $0.2 \mu \mathrm{m}$ syringe filter and dispense the nanosensor solution into a glass vial with screw top cap. The solution should be clear and blue if an aqueous solution that contains less than $10 \mathrm{mM}$ sodium and has a pH less than about 9 is used. Otherwise, it should have no color or be pink.

\section{Determining nanonsensor response}

This method is used to determine the response of nanosensors designed to measure intracellular sodium. Different concentrations are recommended to be used for extracellular sodium concentration nanosensors.

1. Make stock solutions of $10 \mathrm{mM}$ HEPES $(0 \mathrm{Na})$ and $1 \mathrm{M}$ sodium chloride $(\mathrm{NaCl})$ in $10 \mathrm{mM}$ HEPES $(1 \mathrm{M} \mathrm{Na})$ and pH each of these solutions to 7.2 using trizma base. Mix stock solutions of $0 \mathrm{Na}$ and $1 \mathrm{M} \mathrm{Na}$ in $50 \mathrm{ml}$ conical centrifuge tubes to create solutions with: $0,1,5,10,20,50$, $100,200,500,1000 \mathrm{mM} \mathrm{NaCl}$ concentrations.

2. Use an optical bottom 96 well plate to determine the response. Add $100 \mu$ l of each concentration of sodium to 3 wells in a column. This will produce an array of $3 \times 10$ wells.

3. To each well add $100 \mu \mathrm{l}$ of freshly prepared nanosensors and load into a microplate fluorometer. We use a Molecular Devices Spectramax M3.

4. Read each well with the following wavelengths:

\begin{tabular}{|l|l|l|}
\hline Excitation $(\mathbf{n m})$ & Emission $(\mathbf{n m})$ & Cut-Off $(\mathbf{n m})$ \\
\hline 488 & 570 & 530 \\
\hline 488 & 670 & 610 \\
\hline 639 & 680 & 665 \\
\hline
\end{tabular}

The nanosensor intensity at each wavelength is dependent on the sodium concentration. The use of the wavelength depends on the applications. For intracellular slow dynamics measurements, we use $488 \mathrm{~nm} / 570 \mathrm{~nm}$ (excitation/emission) and $488 \mathrm{~nm} / 670 \mathrm{~nm}$ which allow us to ratio the two wavelengths and reduce noise.

5. Traditionally, optode data is converted to a values which are: $\alpha=\left(I-I_{\min }\right) /\left(I_{\max }-I_{\min }\right)$, where $I$ is the intensity at a given sodium concentration, $I_{\min }$ is the lowest intensity in the response measurements, and $I_{\max }$ is the highest intensity in the response measurements. Convert either the ratio of intensity at $570 \mathrm{~nm}$ divided by the intensity at $670 \mathrm{~nm}$ or the intensity at $680 \mathrm{~nm}$ to $\alpha$.

6. Use plotting software, either Excel or Origin are typically used in our lab, to plot $\alpha$ versus the log of the sodium concentration, setting the log of zero sodium concentration to -1. Fit a sigmoidal curve to the response. From the curve, calculate the sodium concentration at $\alpha=0.5$ which represents the concentration at which the sensor optimally responds.

7. Adjust the ratio of $\mathrm{CHIII}, \mathrm{NaIX}$, and NaTFPB in the optode to create nanosensors with optimal response around the sodium concentration of interest. 


\section{Intracellular imaging}

1. For intracellular measurements, the nanosensors are made in $5 \mathrm{mg} / \mathrm{ml}$ glucose in water and microinjected into the cells.

2. Grow or transfer cells to a glass bottom dish or an imaging chamber with a glass coverslip bottom.

3. Incubate the cells in clear media or Tyrode's solution. Mount the imaging chamber or dish onto an epifluorescence microscope. We use a Zeiss LSM 7 confocal microscope.

4. Pull capillary glass with a pipette puller. We use a sutter flaming brown $\mathrm{p}-97$ with $1 \mathrm{~mm}$ OD, $0.78 \mathrm{~mm}$ ID glass, to a final tip diameter around $300-500 \mathrm{~nm}$.

5. Backfill the pipette with nanosensor solution using a syringe and a Hamilton needle, or a microtip loader and a pipette.

6. Mount the pipette into a holder that is attached to a micromanipulator and connected to a pressure controlled injection system. Here we use a Burleigh EXFO PCS 6000 micromanipulator and a medical systems corporation injector.

7. Lower the pipette on top of the cell and into the cytoplasm, sometimes it is necessary to "tap" the manipulator holder to pierce the membrane. Inject the nanosensor solution and quickly withdraw the pipette.

\section{In vivo imaging}

All procedures have been reviewed and approved by Northeastern University's Animal Care and Use Committee.

1. Nanosensors for in vivo, extracellular imaging are made in phosphate buffered saline and adjusted to have an optimal response to sodium at a sodium concentration of $135 \mathrm{mM}$.

2. For the injection set-up and imaging of the fluorescent nanosensors in mice, we use the IVIS Lumina II and its associated anesthesia unit. Disinfect the induction and imaging chambers. Place CD1 nude mice (approximately $20 \mathrm{~g}$ ) in the induction chamber and expose them to isoflurane. The percent of isoflurane and oxygen flowrate administered will vary depending on the species and weight of mice. Wait for the mice to become fully anesthetized.

3. After the mice are anesthetized, remove the mice from the induction chamber and place into the imaging chamber. Keep the mice under anesthesia. For each mouse, disinfect the desired injection areas.

4. Fill a sterile $31 \mathrm{G}$ insulin syringe with $10 \mu \mathrm{L}$ of nanosensors making sure to remove all air bubbles.

5. Using forceps, grasp a small fold of skin along the back of the mouse at the desired injection site. While grasping the skin, insert the syringe into the skin with the bevel facing up and the needle parallel to the skin.

6. Prior to injecting the sensors, pull back on the syringe plunger to ensure the needle is not inserted into a blood vessel. Then, gently move the needle right and left to create a pocket within the skin. This will minimize the back pressure which causes the sensors to leak out of the injection site. Inject the sensors and gently remove the syringe.

7. Gently apply pressure to the injection site and wipe away any solution that may have leaked out of the injection site. This will minimize fluorescence from sensors that are not injected into the skin.

8. Repeats steps 5.4 through 5.7 until the number of desired injection areas is reached. Six injection sites evenly spaced on the left and right side of the back are recommended. For each individual mouse, change needles if the needle becomes difficult to insert into the skin. Needles should not be shared between mice. This will minimize disease transmission or cross-contamination amongst mice.

9. For imaging the sodium fluorescent sensors, we acquire both brightfield and fluorescent images of the mice using filter sets that most closely matched the $640 \mathrm{~nm} / 680 \mathrm{~nm}$ (excitation/emission) spectrum of the nanosensors and that also minimize the autofluorescence of the skin.

\section{Representative Results}

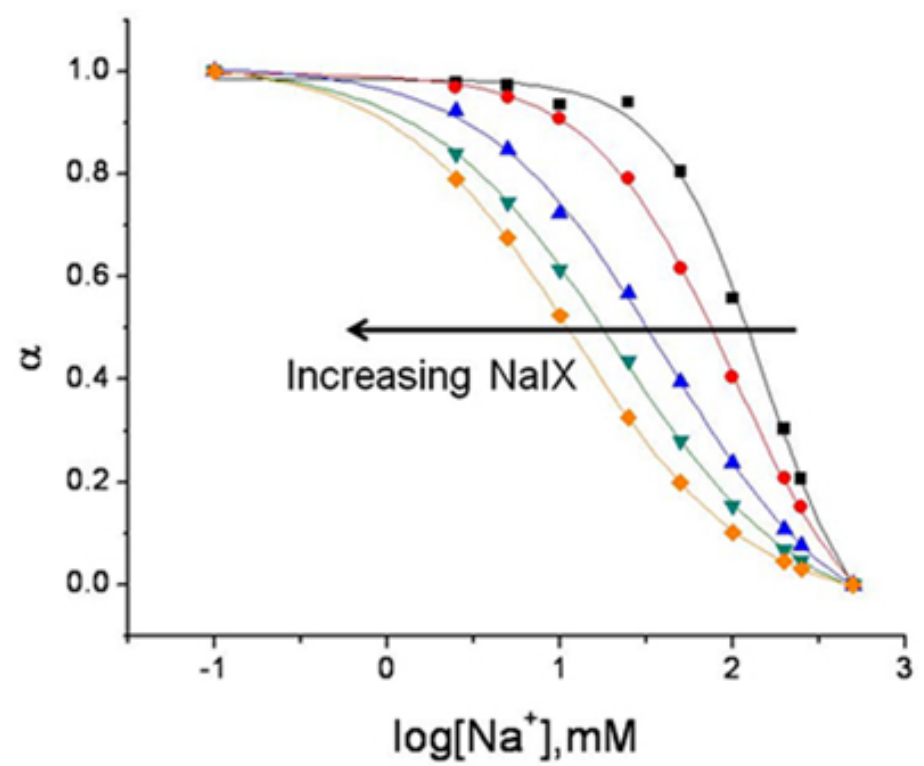

Figure 1. Response curves of five different nanosensor sets to sodium. Each set represents nanosensors from different optode formulations that had the same amount of CHIII, NaTFPB, PVC and DOS but varying amounts of NalX. The data were converted to $\alpha$ values using the $639 / 680$ 
$\mathrm{nm}$ intensities. Data are an average of 3 , error bars omitted for clarity, with a fitted sigmoidal curve using Origin. In this response curve, the maximum concentration of sodium used was $500 \mathrm{mM}$. The $\mathrm{Kd}$ of the sodium nanosensors for sodium ranged from $10 \mathrm{mM}$ (orange diamonds) to about $150 \mathrm{mM}$ (black squares).

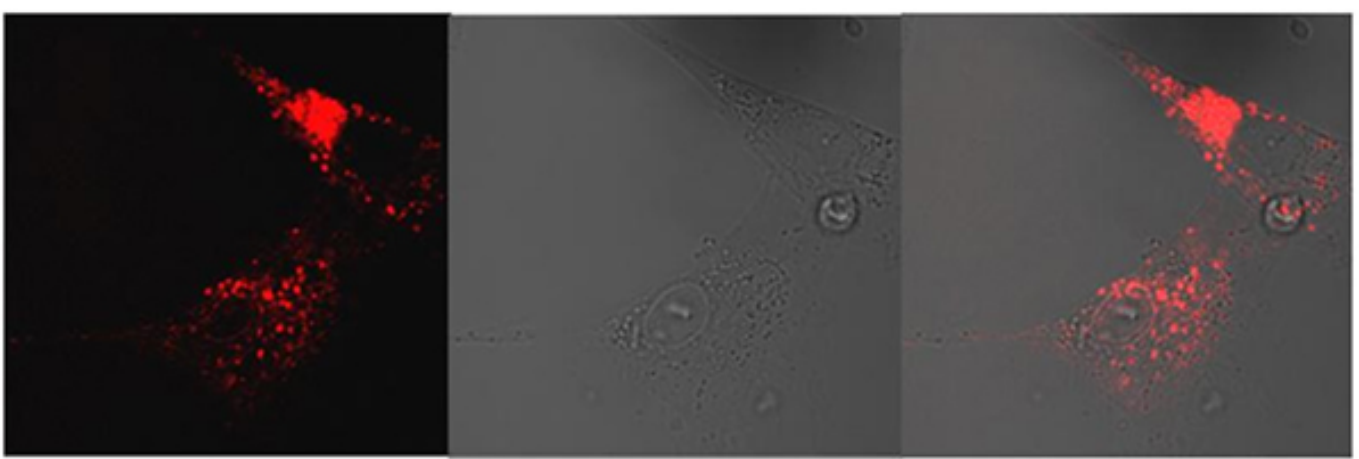

Figure 2. Injected neonatal cardiac myocytes. Cells were cultured on coverglass, mounted on a microscope and injected with sodium nanosensors. Shown are fluorescent $(639 \mathrm{~nm}$ excitation), brightfield, and overlay. Note that some sensor clustering occurs but the cells have normal morphology, sensors have diffused throughout the cytosol and there is no nuclear loading.

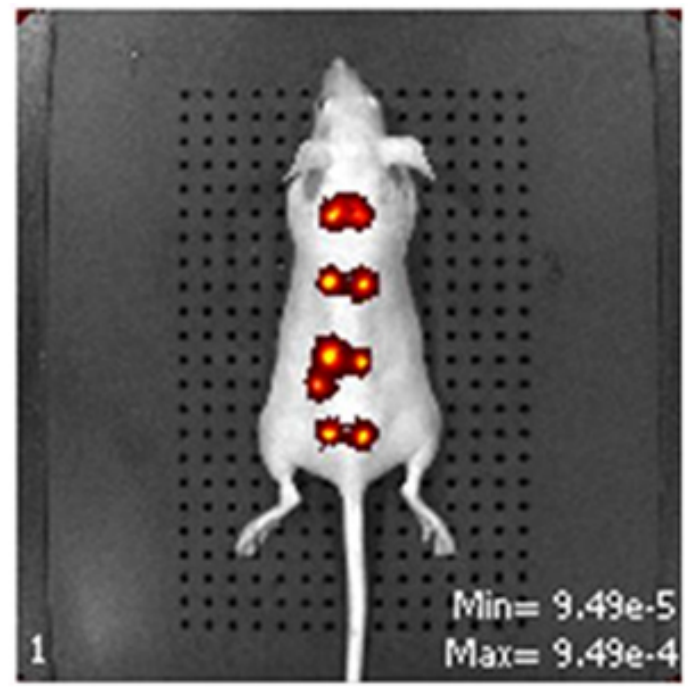

Figure 3. Subcutaneous injection of mice with sodium nanosensors. Nine different injections of sodium nanosensors were made into the subcutaneous space of nude mice. Both bright field and fluorescent images (640/680) are overlaid.

\section{Discussion}

The formation of nanosensors should take no longer than 10 minutes once the optode solution has been made and the PEG lipid dried. Optode can not only be made quickly when needed, but when stored at 4 degrees Celsius, they can be stable for months. We have shown that the nansensors, once formed, are stable in solution for at least a week; however, care must be taken to prevent photobleaching over this time by blocking the nanosensors from light. ${ }^{6}$ While sodium nanosensors were demonstrated here, optodes have been created for most biologically relevant ions such as potassium and chloride. ${ }^{10,12}$ We have also extended this technology to small molecules such as glucose. ${ }^{13}$

In addition to generating nanosensors to monitor different analytes, these nanosensors are amenable to other changes that will make them useful in most biological experiments. For example, the surface coating, in this case poly(ethylene glycol), can easily be changed using any amphiphilic molecule that is water soluble. This enables the possibility of functionalizing these nanoparticles for different applications or targets. The size of the nanoparticles can also be adjusted by altering the surface coating, the sonication intensity or the solvent used.

Calcium fluorescent indicators have been invaluable in determining intracellular calcium signaling; however, no available sodium sensitive dyes have the same ideal characteristics. Optode nanoparticles intended to provide an alternative method for imaging ions intracellularly have been in development for years. ${ }^{14}$ We have built upon this previous research to create nanosensors specific for intracellular sodium imaging that will hopefully provide a means to understand how sodium signaling affects cellular function and alterations in signaling which lead to certain diseases.

The use of fluorescent nanosensors in vivo offers a real-time minimally-invasive alternative for monitoring analytes over other methods such as blood draws. ${ }^{3}$ Sodium and glucose nanosensors based on the technology above have been shown to track changes in sodium and glucose, respectively in vivo. ${ }^{3,13}$ However, there currently exist limitations to this method as a monitoring tool. For example, the sensors must contain a fluorophore with a spectrum sufficiently shifted towards the near infrared red in order to minimize background autofluorescence from the skin. ${ }^{15}$ 
Second, the current injection technique does not produce uniform injection of the nanosensors which may be caused by such errors as variation in injection depth. Despite these limitations, the development of these fluorescent nanosensors and their successful demonstration could make these sensors an invaluable research tool and method for monitoring patient health.

\section{Disclosures}

No conflicts of interest declared.

\section{Acknowledgements}

JMD and MKB are funded through the IGERT Nanomedicine Science and Technology program at Northeastern University (funding from NCl and NSF grant DGE- 0504331). This work was also funded by National Institutes of Health National Institute of General Medical Sciences Grant R01 GM084366. We also thank Saumya Das and Anthony Rosenzweig of BIDMC in Boston for providing cardiac myocytes and Kevin Cash for his contribution in the animal protocol development.

\section{References}

1. Adrogue, H. J., \& Madias, N. E. Hyponatremia. N Engl J Med 342, 1581-1589 (2000).

2. Kim, D. Y. et al. BACE1 regulates voltage-gated sodium channels and neuronal activity. Nat Cell Biol 9, 755-764, doi:ncb1602 [pii] 10.1038/ ncb1602 (2007).

3. Dubach, J. M., Lim, E., Zhang, N., Francis, K. P., \& Clark, H. in vivo sodium concentration continuously monitored with fluorescent sensors. Integr Biol (Camb), doi:10.1039/c0ib00020e (2010).

4. Minta, A., \& Tsien, R. Y. Fluorescent indicators for cytosolic sodium. J Biol Chem 264, 19449-19457 (1989).

5. Dubach, J. M., Harjes, D. I., \& Clark, H. A. Ion-selective nano-optodes incorporating quantum dots. Journal of the American Chemical Society 129, 8418-+, doi:Doi 10.1021/Ja072522i (2007)

6. Dubach, J. M., Harjes, D. I., \& Clark, H. A. Fluorescent ion-selective nanosensors for intracellular analysis with improved lifetime and size. Nano Letters 7, 1827-1831, doi:Doi 10.1021/NI0707860 (2007).

7. Schaffar, B., \& Wolfbeis, O. A sodium-selective optrode. Mikrochim Acta III, 109 (1989).

8. Seiler, K. et al. Characterization of sodium-selective optode membranes based on neutral ionophores and assay of sodium in plasma. Clin Chem 37, 1350-1355 (1991)

9. Zhujun, Z., Mullin, J., \& Seitz, W. Optical sensor for sodium based on ion-pair extraction and fluorescence. Anal Chim Acta 184, 251 (1986).

10. Bakker, E., Buhlmann, P., \& Pretsch, E. Carrier-Based lon-Selective Electrodes and Bulk Optodes. 1. General Characteristics. Chem Rev 97, 3083-3132, doi:cr940394a [pii] (1997).

11. Dubach, J. M., Das, S., Rosenzweig, A., \& Clark, H. A. Visualizing sodium dynamics in isolated cardiomyocytes using fluorescent nanosensors. Proceedings of the National Academy of Sciences of the United States of America 106, 16145-16150, doi:DOI 10.1073/ pnas.0905909106 (2009).

12. Harjes, D. I., Dubach, J. M., Rosenzweig, A., Das, S., \& Clark, H. A. Ion-Selective Optodes Measure Extracellular Potassium Flux in Excitable Cells. Macromolecular Rapid Communications 31, 217-221, doi:DOI 10.1002/marc.200900297 (2010).

13. Balaconis, M. K., Billingsley, K.L., Dubach, J.M., \& Clark, H.A. in Pittsburgh Conference on Analytical Chemistry and Applied Spectroscopy.

14. Park, E. J., Brasuel, M., Behrend, C., Philbert, M. A., \& Kopelman, R. Ratiometric optical PEBBLE nanosensors for real-time magnesium ion concentrations inside viable cells. Anal Chem 75, 3784-3791 (2003).

15. Frangioni, J. V. in vivo Near-Infrared Fluorescence Imaging. Curr Op Chem Biol 7, 626-634 (2003). 